\title{
Comparison between self-reported physical activity (IPAQ-SF) and pedometer among overweight and obese women in the MyBFF@home study
}

\author{
Mohamad Hasnan Ahmad ${ }^{1 *}$, Ruhaya Salleh', Noor Safiza Mohamad Nor ${ }^{1}$, Azli Baharuddin', \\ Wan Shakira Rodzlan Hasani', Azahadi Omar ${ }^{1}$, Ahmad Taufik Jamil², Mahenderan Appukutty ${ }^{3}$, \\ Wan Abdul Manan Wan Muda ${ }^{4}$ and Tahir Aris ${ }^{1}$
}

\begin{abstract}
Background: Several methods have been developed to determine a person's physical activity level. However, there is limited evidence in determining whether someone is physically active or not. This study aims to determine the level of physical activity and to compare the usage of short version International Physical Activity Questionnaire (IPAQ-SF) and pedometer among overweight and obese women who were involved in the My Body is Fit and Fabulous at home (MyBFF@home) study.

Methods: Baseline and sixth month data from the MyBFF@home study were used for this purpose. A total of 169 of overweight and obese respondents answered the IPAQ-SF and were asked to use a pedometer for 7 days. Data from IPAQ-SF were categorised as inactive and active while data from pedometer were categorised as insufficiently active and sufficiently active by standard classification. Data on sociodemographic and anthropometry were also obtained. Cohen's kappa was applied to measure the agreement of IPAQ-SF and pedometer in determining the physical activity level. Pre-post cross tabulation table was created to evaluate the changes in physical activity over 6 months.

Results: From 169 available respondents, 167 (98.8\%) completed the IPAQ-SF and 107 (63.3\%) utilised the pedometer. A total of 102 (61.1\%) respondents were categorised as active from the IPAQ-SF. Meanwhile, only 9 (8.4\%) respondents were categorised as sufficiently active via pedometer. Cohen's $\mathrm{k}$ found there was a poor agreement between the two methods, $K=0.055, p>0.05$. After sixth months, there was $+9.4 \%$ increment in respondents who were active when assessed by IPAQ-SF but $-1.3 \%$ reductions for respondents being sufficiently active when assessed by pedometer. McNemar's test determined that there was no significant difference in the proportion of inactive and active respondents by IPAQ-SF or sufficiently active and insufficiently active by pedometer from the baseline and sixth month of intervention.
\end{abstract}

Conclusion: The IPAQ-SF and pedometer were both able to measure physical activity. However, poor agreement between these two methods were observed among overweight and obese women.

Keywords: Physical activity, International physical activity questionnaire (IPAQ-SF), Pedometer, Obese women, Malaysia

\footnotetext{
* Correspondence: hasnan.ahmad@moh.gov.my

${ }^{1}$ Institute for Public Health, National Institutes of Health, Ministry of Health

Malaysia, Kuala Lumpur, Malaysia

Full list of author information is available at the end of the article
}

(c) The Author(s). 2018 Open Access This article is distributed under the terms of the Creative Commons Attribution 4.0 International License (http://creativecommons.org/licenses/by/4.0/), which permits unrestricted use, distribution, and reproduction in any medium, provided you give appropriate credit to the original author(s) and the source, provide a link to the Creative Commons license, and indicate if changes were made. The Creative Commons Public Domain Dedication waiver (http://creativecommons.org/publicdomain/zero/1.0/) applies to the data made available in this article, unless otherwise stated. 


\section{Background}

Physical activity is defined as the body movement produced by skeletal muscles that results in energy expenditure [1]. However, the level of physical activity differs from person to person depending on his or her personal choice, which is in turn largely subject to many internal and external factors [2].

Nowadays, the prevalence of overweight and obesity is rising around the world and has become a global issue. Modernisation and advancement of transportation is believed to reduce the physical activity of people and contribute towards this epidemic [3]. The excess calories due to physical inactivity will be stored in the body as fat and if it continues, he or she will have excess body fat which will increase their body weight [4].

Based on the most recent findings from the National Health \& Morbidity Survey (NHMS) 2015, more women in Malaysia were categorised as overweight and obese compared to men [5]. Excess body weight among women has always been a big concern either for their health or body image. Being physically active is highly recommended in order for women to reduce their body weight other than reducing calorie intake [6]. However, the way women are physically active might be different from men, as majority of women in Malaysia are housewives who are responsible for all household chores [7]. They may have more time, but most of their time are spent at home being sedentary or doing housework.

The assessment of physical activity is complex and troublesome to get an accurate estimation. However, the use of suitable methods in determining physical activity level is important to ensure accurate information on physical activity is obtained to produce effective intervention programmes. Several methods have been developed to determine physical activity levels. These methods can be divided into two categories. The first one is self-reported measurement such as the Global Physical Activity Questionnaire (GPAQ), the International Physical Activity Questionnaire-Short Form (IPAQ-SF) and physical activity diary. The second one is a direct measure of physical activity which includes calorimeter, accelerometer and pedometer usage [8].

However, from the available methods, there is limited evidence in determining whether someone is physically active or not, especially among women with excess body weight. This is because, previous studies have reported that there was high potential of bias on physical activity assessment especially by self-reported methods and among certain group of people [9]. Therefore, this paper aimed to determine the level of physical activity, and to compare the usage of a self-reported measure of physical activity which is the International Physical Activity Questionnaire-Short Form (IPAQ-SF) and the direct measure using pedometer among overweight and obese women living in low-cost flats.

\section{Methods}

Baseline and sixth month data were obtained from the My Body is Fit \& Fabulous at home (MyBFF@home) study. This was a community-based intervention study to combat obesity among overweight and obese women in the Klang Valley, which includes the central area of Kuala Lumpur and its adjoining cities and towns in the state of Selangor. The methodology and the details of the MyBFF@home study have been reported elsewhere [10].

\section{Variables used}

Data on sociodemographic, anthropometry and physical activity assessment by using Short Form of International Physical Activity Questionnaire (IPAQ-SF) and pedometer were obtained. Socio demographic data included age, ethnicity, education level, marital status, number of children and household income. Meanwhile, data for anthropometry were weight, height and waist circumference. Data on weight and height were used to calculate Body Mass Index (BMI) and recoded based on World Health Organization 1998 cut-off points. Data for waist circumference were recoded according to cut-off recommendations for Asian populations [11].

Respondents were asked to complete the IPAQ-SF. The IPAQ-SF records the last 7 day recall for four intensity levels of physical activity which is vigorous-intensity activity, moderate-intensity activity, walking, and sitting. From IPAQ-SF, data were converted to Metabolic Equivalent minutes per week (MET-min/week) using the published formulation by Ainsworth et al. [12]. Compendium average MET score (Walking = 3.3 METs, Moderate Physical Activity $=4.0 \mathrm{MET}$ and Vigorous Physical Activity = 8.0 METs) [13].

Respondents who met these following criteria, which is $\geq 3$ days of vigorous activity of at least 20 min per day, or $\geq 5$ days of moderate-intensity activity and walking of at least 30 min per day, or $\geq 5$ days of any combination of walking, moderate-intensity and vigorous intensity activities achieving a minimum of at least 600 MET-min/week, or Vigorous-intensity activity on at least 3 days and accumulating at least 1500 MET minutes/week or $\geq 7$ days of any combination of walking, moderate-intensity and vigorous intensity activities achieving a minimum of at least 3000 MET-minutes/ week, were considered as active. Those respondents not meeting the above criteria were categorised as inactive [14]. Meanwhile, data on steps count from pedometer were converted into two categories of physical activity; those with less than 10,000 steps per day as insufficiently active and those with $\geq 10,000$ steps per day as sufficiently active [15]. 


\section{Data analysis}

The data was analysed using IBM SPSS version 23 for Windows (SPSS Inc., Chicago, IL, USA). Data was harmonized and recoded according to the study objective. Descriptive statistics were used for describing the characteristics of the respondent. The two by two cross-tabulation table was created and Cohen's kappa was calculated to measure the level of agreement between IPAQ and pedometer in the classification of physical activity level using baseline data. The McNemar test was carried out to determine if there were differences on a dichotomous dependent variable which included physical activity levels by IPAQ-SF and pedometer from baseline to sixth month of the intervention.

\section{Results}

From the 169 available respondents, 167 (98.8\%) completed the IPAQ-SF and 107 (63.3\%) utilised the pedometer. The average steps count from pedometer was 5196 steps per day with minimum steps recorded as only 377 and the maximum being 19,303.

By IPAQ-SF, 61.1\% respondents were categorised as active. There were more physically active respondents in the 40-49 years age group, Malay ethic group, women who had secondary and high education level, women who were married, had 3-4 number of children, those with household income more than RM2,501.00, those in pre-obesity BMI category and with no abdominal obesity by waist circumference classification. About $8.4 \%$ respondent were sufficiently active from pedometer. There were more sufficiently active respondents from the younger age group (18-29 years old), Chinese ethnic group, with minimum secondary education, unmarried, with less than 2 children, income between RM1501.00 - RM2500.00, being obese II and with no abdominal obesity by waist circumference classification as shown in Table 1.

Cohen's $\mathrm{k}$ was used to determine if there was an agreement between the two methods in determining physical activity levels among the respondents. There was a poor agreement between these two methods, Cohen's $\mathrm{K}=0.055$, $p>0.05$, as shown in Table 2 .

Over the course of sixth months, there was some increase in the number respondents who were active when assessed by IPAQ-SF but a slight reduction for respondents being sufficiently active when assessed by pedometer. The exact McNemar's test showed that there was no significant difference in the proportion of respondents inactive and active by IPAQ-SF or sufficiently active and insufficiently active by pedometer from the baseline and sixth month of intervention as shown in Table 3.

\section{Discussion}

This recent study reported that levels of physical activity among overweight and obese women who live in low-cost flats in Klang Valley was higher when measured using the self-reported measure (IPAQ-SF) compared to direct measurement by pedometer. Previous study comparing subjective (IPAQ) and objective (accelerometer) measure of physical activity also found almost similar finding, whereby IPAQ is more likely to overestimate actual physical activity by its limited ability to classify adults into low and high categories of physical [14]. Over the 6 months intervention in MyBFF@home program, the level of high physical activity assessed by pedometer saw a slightly drop compared to the IPAQ-SF. This is because the type of intervention in MyBFF@home mainly focuses on 12 step exercise using pillow dumbbell exercise, which activity is not capture by pedometer [10].

The IPAQ-SF development was premised on the need to develop international population measurement to assess 'total physical activity' and categorize respondents into various levels of physical activity [15]. It relies on ability to recall and honesty of the respondents to report the physical activity which they have engaged in over the past 7 days [16]. A previous study also reported that the IPAQ-SF does not appear to be a good indicator of individual physical activity behaviour especially among older adults but is better suited for larger population-based samples [17].

The pedometer on the other hand, does not rely on self-recall of physical activity or subjective assessment of exercise intensity, in contrast to physical activity questionnaires [18]. This device directly senses the body motion and counts the footsteps [19]. Many previous studies reported that pedometers are a good proxy to physical activity because it reduces the subjectivity inherent in survey methods. It can be applied to large groups of individuals and also allows results from research studies to be readily translated into physical activity levels $[18,20]$. However, the limitation of pedometers is that it does not measure all types of physical activity, and it is acknowledged that they do not capture swimming, cycling, and weight lifting [21]. Studies also found inverse trends in physical activity among obesity status. Those who are pre-obese were found to be more active when assessed by self-reported measure but insufficiently active when assessed by direct measure [22, 23]. Conversely, those who were obese type II were found to be more inactive when assessed by IPAQ-SF but sufficiently active when assessed by pedometer.

The findings coincide with a systematic review conducted in 2008 which reported correlations between self-reported and direct measures were generally uncertain and it can be negatively or positively correlated, ranging from -0.71 to 0.96 . The same systematic review also concluded that the self-reported measures of physical activity were both higher and lower than directly 
Table 1 Characteristics of the respondents and status of physical activity based on IPAQ-SF and pedometer, n (\%)

\begin{tabular}{|c|c|c|c|c|c|}
\hline \multirow[t]{3}{*}{ Variable } & \multirow{3}{*}{$\begin{array}{l}\text { Number of } \\
\text { respondents }\end{array}$} & \multicolumn{4}{|c|}{ Physical activity level } \\
\hline & & \multicolumn{2}{|l|}{ IPAQ-SF } & \multicolumn{2}{|l|}{ Pedometer } \\
\hline & & Inactive & Active & Insufficiently active & Sufficiently active \\
\hline Total respondents & $169(100)$ & 65 (38.9) & $102(61.1)$ & $98(91.6)$ & $9(8.4)$ \\
\hline \multicolumn{6}{|l|}{ Age (years) } \\
\hline $18-29$ & $14(8.3)$ & $6(42.9)$ & $8(57.1)$ & $7(87.5)$ & $1(12.5)$ \\
\hline $30-39$ & $48(28.4)$ & $20(41.7)$ & $28(58.3)$ & $26(92.9)$ & $2(7.1)$ \\
\hline $40-49$ & $72(42.6)$ & $21(29.6)$ & $50(70.4)$ & $44(89.8)$ & $5(10.2)$ \\
\hline$\geq 50$ & $35(20.7)$ & $18(52.9)$ & $16(47.1)$ & $21(95.5)$ & $1(4.5)$ \\
\hline \multicolumn{6}{|l|}{ Ethnicity } \\
\hline Malay & $143(84.6)$ & $54(38.3)$ & $87(61.7)$ & $90(91.8)$ & $8(8.2)$ \\
\hline Chinese & $5(3.0)$ & $2(40.0)$ & $3(60.0)$ & $2(66.7)$ & $1(33.3)$ \\
\hline Indian & $18(10.7)$ & $7(38.9)$ & $11(61.1)$ & $4(100.0)$ & $0(0.0)$ \\
\hline Others & $3(1.8)$ & $2(66.7)$ & $1(33.3)$ & $2(100.0)$ & $0(0.0)$ \\
\hline \multicolumn{6}{|l|}{ Education } \\
\hline Primary and lower & $29(17.3)$ & $16(55.2)$ & $13(44.8)$ & $16(94.1)$ & $1(5.9)$ \\
\hline Secondary and higher & $139(82.7)$ & $49(35.8)$ & $88(64.2)$ & $82(91.1)$ & $8(8.9)$ \\
\hline \multicolumn{6}{|l|}{ Marital status } \\
\hline Married & $153(90.5)$ & $57(37.5)$ & $95(62.5)$ & $88(92.6)$ & $7(7.4)$ \\
\hline Not married/ widowed/separated & $16(9.5)$ & $8(53.3)$ & $7(46.7)$ & $10(83.3)$ & $2(16.7)$ \\
\hline \multicolumn{6}{|l|}{ Number of children } \\
\hline$\leq 2$ & $38(22.6)$ & $17(44.7)$ & $21(55.3)$ & $18(85.7)$ & $3(14.3)$ \\
\hline $3-4$ & $82(48.8)$ & $26(32.1)$ & $55(67.9)$ & $47(90.4)$ & $5(9.6)$ \\
\hline$\geq 5$ & $48(28.6)$ & $21(44.7)$ & $26(55.7)$ & $33(97.1)$ & $1(2.9)$ \\
\hline \multicolumn{6}{|l|}{ Household income } \\
\hline$\leq \mathrm{RM} 1500.00$ & $80(48.2)$ & $38(48.7)$ & $40(51.3)$ & $43(95.6)$ & $2(4.4)$ \\
\hline RM 1501.00-2500.00 & $54(32.5)$ & $19(35.2)$ & $35(64.8)$ & $34(87.2)$ & $5(12.8)$ \\
\hline$\geq$ RM 2501.00 & $32(18.3)$ & $7(21.9)$ & $25(78.1)$ & $20(90.1)$ & $2(9.9)$ \\
\hline \multicolumn{6}{|l|}{ Obesity status } \\
\hline Pre-obesity & $73(43.2)$ & $27(37.5)$ & $45(62.5)$ & $40(93.2)$ & $3(6.8)$ \\
\hline Obese I & $58(34.3)$ & $22(38.6)$ & $35(61.4)$ & $33(91.7)$ & $3(8.3)$ \\
\hline Obese II & $38(22.5)$ & $16(42.1)$ & $22(57.9)$ & $27(88.9)$ & $9(11.1)$ \\
\hline \multicolumn{6}{|l|}{ Waist circumference } \\
\hline$\leq 80 \mathrm{~cm}$ & $12(7.1)$ & $2(16.7)$ & $10(83.3)$ & $7(87.5)$ & $1(12.5)$ \\
\hline$>80 \mathrm{~cm}$ & $157(92.9)$ & $63(40.6)$ & $92(59.4)$ & $91(91.9)$ & $8(8.1)$ \\
\hline
\end{tabular}

Table 2 Measure of agreement between IPAQ-SF and pedometer on physical activity classification n (\%)

\begin{tabular}{|c|c|c|c|c|c|}
\hline & & \multicolumn{2}{|l|}{ Pedometer } & \multirow{2}{*}{$\begin{array}{l}\text { Cohen's } \\
\text { K }\end{array}$} & \multirow[t]{2}{*}{$p$-value } \\
\hline & & Insufficiently active & Sufficiently active & & \\
\hline \multirow[t]{2}{*}{ IPAQ-SF } & Inactive & $42(95.5)$ & $2(4.5)$ & 0.055 & 0.229 \\
\hline & Active & $56(88.9)$ & $7(11.1)$ & & \\
\hline
\end{tabular}


Table 3 Change in physical activity level by IPAQ-SF and pedometer from baseline to sixth month $\mathrm{n}$ (\%)

\begin{tabular}{|c|c|c|c|c|c|}
\hline \multirow[t]{2}{*}{ Test } & \multirow[t]{2}{*}{ Category } & \multicolumn{2}{|c|}{ Intervention period } & \multirow{2}{*}{$\begin{array}{l}\text { Percent } \\
\text { changes }\end{array}$} & \multirow{2}{*}{$\begin{array}{l}\text { McNema } \\
p \text {-value }\end{array}$} \\
\hline & & Baseline & Sixth month & & \\
\hline \multirow[t]{2}{*}{ IPAQ-SF } & Inactive & 65 (38.9) & $36(29.5)$ & -9.4 & 0.067 \\
\hline & Active & $102(61.1)$ & $86(70.5)$ & +9.4 & \\
\hline \multirow[t]{2}{*}{ Pedometer } & Insufficient active & $98(91.6)$ & 39 (92.9) & +1.3 & 0.999 \\
\hline & Sufficient active & $9(8.4)$ & $3(7.1)$ & -1.3 & \\
\hline
\end{tabular}

$p<0.05$, significant for McNemar test

measured levels of physical activity, which poses a problem for both reliance on self-reported measures and for attempts to correct for self-reported - directly measured differences [8].

There is also a systematic review on validity of IPAQ-SF reporting that the correlation between the IPAQ-SF and objective measures of activity or fitness was lower than the acceptable standard in the large majority of studies. Furthermore, the IPAQ-SF typically overestimated physical activity as measured by objective criterion by an average of $84 \%$. Hence, the evidence to support the use of the IPAQ-SF as an indicator of relative or absolute physical activity is weak [24].

A critical appraisal paper on assessment of physical activity published in 2009 reported that doubly labelled water method was the gold standard in assessing physical activity. However, this method gives high interference to the respondent, is very expensive and the data does not provide specific information on daily physical activity. Questionnaires which use self-reported methods show a low reliability and validity but can be adequately applied as an activity-ranking instrument [25]. Pedometer or accelerometer was also a good instrument for assessing physical activity but it needs to be upgraded so that it can provide not just step count data, but also information on body posture and activity recognition to allow objective assessment of subjects' habitual activities, options for a healthy change, and effects of the follow-up of any changes [25].

There are several strengths and limitations in this study. The response rate for IPAQ-SF was good but the response rate for pedometer was low. However, it is still adequate to perform statistical analysis at the baseline. The level of motivation and awareness among the participants could be one of the barriers to using the pedometer every day. Almost half of the respondents have more than five children and they spent most of their time doing housework at home or attending to their children. The other limitation is the distribution of the respondents is not equal across sociodemographic variables. This imbalance does not allow reliable inferential statistical tests to be applied to the data especially for the sixth month. Thus, the results should be interpreted with caution.

\section{Conclusion}

In summary, the physical activity status among overweight and obese women was high using the self-report method, IPAQ-SF as compared to the direct method using pedometer. Overall, we found that physical activity levels among overweight and obese women who are living in low-cost flats in Klang Valley to be low. It was also found that there is a lack of agreement in determining physical activity level by IPAQ-SF and pedometer. Therefore, the usage of the IPAQ-SF or pedometer in measuring physical activity must be focused on the objective since both methods measure different sides of physical activity.

\section{Abbreviations}

GPAQ: Global Physical Activity Questionnaire; IPAQ-SF: International Physical Activity Questionnaire -Short Form; MyBFF@home: My Body is Fit and

Fabulous at Home; NHMS: National Health and Morbidity Survey

\section{Acknowledgements}

The authors would like to thank the Director General of Health Malaysia for the permission to publish this paper. The authors would also like to thank all research team members from various institutions and Dr. Wan Nazaimoon Wan Mohamud (former MyBFF project coordinator) for their technical support. Special thanks to all the participants in the MyBFF@home study, for their full cooperation and dedication.

\section{Funding}

Publication of this article was sponsored by the Ministry of Health Malaysia.

\section{Availability of data and materials}

The dataset that support the findings of this article belong to the MyBFF@home study. At present, the data are not publicly available but can be obtained from the authors upon reasonable request and with the permission from the Director General of Health, Malaysia.

\section{About this supplement}

This article has been published as part of BMC Women's Health Volume 18 Supplement 1, 2018: My Body is Fit and Fabulous at home (MyBFF@home). The full contents of the supplement are available online at https://bmc womenshealth.biomedcentral.com/articles/supplements/volume-18supplement-1.

\section{Authors' contributions}

MHA and RS performed the statistical analysis and drafted the manuscript. NSMN responsible for the concept, design the project and supervision. $A B$, WSRH and MA contributed to review and editing this manuscript. AO, ATJ, WAMWM and TA contributed to interpretation of the results, and preparation of the manuscript. All authors have read and approved the final manuscript.

\section{Ethics approval and consent to participate}

Ethical approval for the MyBFF@home study was obtained from the Medical Research Ethic Committee (MREC) Malaysia (Research registration number: NMRR-13-726-16,391). Informed written consent was taken from all respondents at the beginning of the study. 


\section{Consent for publication}

Not applicable.

\section{Competing interests}

The authors declare that they have no competing interests.

\section{Publisher's Note}

Springer Nature remains neutral with regard to jurisdictional claims in published maps and institutional affiliations.

\section{Author details}

'Institute for Public Health, National Institutes of Health, Ministry of Health Malaysia, Kuala Lumpur, Malaysia. ${ }^{2}$ Population Health and Preventive Medicine, Faculty of Medicine, Universiti Teknologi Mara (UiTM), Sungai Buloh Campus, Jalan Hospital, Sungai Buloh, Selangor Darul Ehsan, Malaysia. ${ }^{3}$ Faculty of Sport Science \& Recreation, Universiti Teknologi Mara (UiTM), Shah Alam Campus, Shah Alam, Selangor Darul Ehsan, Malaysia. ${ }^{4}$ Center for Southeast Asian Studies, Kyoto University, Kyoto, Japan.

\section{Published: 19 July 2018}

\section{References}

1. Caspersen CJ, Powell KE, Christenson GM. Physical activity, exercise, and physical fitness: definitions and distinctions for health-related research. Public Health Rep. 1985;100:126.

2. Booth SL, Sallis JF, Ritenbaugh C, Hill JO, Birch LL, Frank LD, et al. Environmental and societal factors affect food choice and physical activity: rationale, influences, and leverage points. Nut Rev. 2001;59:21-36.

3. Katzmarzyk PT, Mason C. The physical activity transitions. J Phys Act Health. 2009:6:269-80.

4. Bordo S. Unbearable weight: Feminism, Western culture, and the body. Berkeley: Univ of California Press; 2004. p. 481-82.

5. Institute for Public Health. National Health \& Morbidity Survey 2015 (NHMS 2015). Vol. II: non-communicable diseases. Kuala Lumpur: Ministry of Health Malaysia; 2015.

6. Hamilton MT, Healy GN, Dunstan DW, Zderic TW, Owen N. Too little exercise and too much sitting: inactivity physiology and the need for new recommendations on sedentary behavior. Curr Cardiovasc Risk Rep. 2008;2: 292-8.

7. Teh CH, Lim KK, Chan YY, Lim KH, Azahadi O, Akmar AH, et al. The prevalence of physical activity and its associated factors among Malaysian adults: findings from the National Health and morbidity survey 2011. Pub Health. 2014;128:416-23.

8. Prince SA, Adamo KB, Hamel ME, Hardt J, Gorber SC, Tremblay M. A comparison of direct versus self-report measures for assessing physical activity in adults: a systematic review. Int J Behav Nutr Phys Act. 2008;5:56.

9. Sallis JF, Saelens BE. Assessment of physical activity by self-report: status, limitations, and future directions. Res Q Exerc Sport. 2000;71:1-4.

10. Mohamad Nor NS, Ambak R, Omar MA, Shahar S, Abdul Aziz NS, Muhamad Yusuff MF, et al. Methodology of the my body is fit and fabulous at home (MyBFF@ home): an intervention study to combat obesity among housewives in Malaysia. J Womens Health Issues Care. 2016;5:2.

11. World Health Organization. Waist circumference and waist-hip ratio: Report of a WHO expert consultation. 2011.

12. Hagströmer M, Oja P, Sjöström M. The international physical activity questionnaire (IPAQ): a study of concurrent and construct validity. Pub Health Nutr. 2006;9:755-62.

13. Ainsworth BE, Haskell WL, Herrmann SD, Meckes N, Bassett DR, Tudor-Locke C, et al. 2011 compendium of physical activities: a second update of codes and MET values. Med Sci Sports Exerc. 2011;43:1575-81.

14. Hagstromer M, Ainsworth BE, Oja P, Sjostrom M. Comparison of a subjective and an objective measure of physical activity in a population sample. J Phys Act Health. 2010;4:541-50.

15. IPAQ Research Committee. Guidelines for data processing and analysis of the International Physical Activity Questionnaire (IPAQ)-short and long forms. 2005. https://sites.google.com/site/theipaq/scoring-protocol. Accessed 26 Feb 2018.

16. Wilde BE, Sidman CL, Corbin CB. A 10,000-step count as a physical activity target for sedentary women. Res Q Exerc Sport. 2011;72:411-4.

17. Tudor-Locke C, Bassett DR. How many steps/days are enough? Sports Med. 2004;34:1-8
18. Bauman A, Ainsworth BE, Bull F, Craig CL, Hagströmer M, Sallis JF, et al. Progress and pitfalls in the use of the international physical activity questionnaire (IPAQ) for adult physical activity surveillance. J Phys Act Health. 2009:6:5-8.

19. Warren JM, Ekelund U, Besson H, Mezzani A, Geladas N, Vanhees L. Assessment of physical activity-a review of methodologies with reference to epidemiological research: a report of the exercise physiology section of the European Association of Cardiovascular Prevention and Rehabilitation. Eur J Cardiovasc Prev Rehabil. 2010;17:127-39.

20. Grimm EK, Swartz AM, Hart T, Miller NE, Strath SJ. Comparison of the IPAQshort form and accelerometry predictions of physical activity in older adults. J Aging Phys Act. 2012;20:64-79.

21. Tudor-Locke C, Williams JE, Reis JP, Pluto D. Utility of pedometers for assessing physical activity. Sports Med. 2002;32:795-808.

22. Bassett DR, Strath SJ. Use of pedometers to assess physical activity. Physical activity assessments for health-related research; 2002. p. 163-77.

23. Welk G. Physical activity assessments for health-related research. Human Kinetics. 2002. p.105-10.

24. Lee PH, Macfarlane DJ, Lam TH, Stewart SM. Validity of the international physical activity questionnaire short form (IPAQ-SF): a systematic review. Int J Behav Nutr Phys Act. 2011;8:115.

25. Westerterp KR. Assessment of physical activity: a critical appraisal. Eur J Appl Physiol. 2009;105:823-8.

\section{Ready to submit your research? Choose BMC and benefit from:}

- fast, convenient online submission

- thorough peer review by experienced researchers in your field

- rapid publication on acceptance

- support for research data, including large and complex data types

- gold Open Access which fosters wider collaboration and increased citations

- maximum visibility for your research: over $100 \mathrm{M}$ website views per year

At BMC, research is always in progress.

Learn more biomedcentral.com/submissions 